\title{
Novel mutations in LRP6 highlight the role of WNT signaling in tooth agenesis
}

\author{
Charlotte W. Ockeloen, MD¹, Kriti D. Khandelwal, MSc², Karoline Dreesen, DDS ${ }^{2,3}$, \\ Kerstin U. Ludwig, $\mathrm{PhD}^{4}$, Robert Sullivan, BSc${ }^{5}$, Iris A.L.M. van Rooij, $\mathrm{PhD}^{6}$, Michelle Thonissen, $\mathrm{MSc}^{2}$, \\ Steven Swinnen, DDS ${ }^{3}$, Milien Phan, MSc${ }^{2}$, Federica Conte, $\mathrm{MSc}^{7}$, Nina Ishorst, $\mathrm{MSc}^{4}$, \\ Christian Gilissen, PhD ${ }^{1}$, Laury Roa Fuentes, $\mathrm{MSc}^{2}$, Maartje van de Vorst, BSc${ }^{1}$, Arjen Henkes, $\mathrm{BSc}^{1}$, \\ Marloes Steehouwer, BSc${ }^{1}$, Ellen van Beusekom, BSc${ }^{1}$, Marjon Bloemen, $\mathrm{MSc}^{2}$, \\ Bruno Vankeirsbilck, BSc${ }^{8}$, Stefaan Bergé, MD, PhD ${ }^{9}$, Greet Hens, MD, $\mathrm{PhD}^{10}$, \\ Joseph Schoenaers, MD ${ }^{10}$, Vincent Vander Poorten, MD, PhD ${ }^{10}$, Jasmien Roosenboom, $\mathrm{MSc}^{10}$, \\ An Verdonck, DDS, PhD2,10, Koen Devriendt, MD, PhD ${ }^{10,11}$, Nel Roeleveldt, $\mathrm{PhD}^{6}$, \\ Shalini N. Jhangiani, MSc'12, Lisenka E.L.M. Vissers, PhD1', James R. Lupski, MD, PhD ${ }^{12-15}$, \\ Joep de Ligt, PhD ${ }^{16}$, Johannes W. Von den Hoff, PhD², Rolph Pfundt, PhD', \\ Han G. Brunner, MD, PhD ${ }^{1}$, Huiqing Zhou, PhD ${ }^{1,7}$, Jill Dixon, $\mathrm{PhD}^{5}$, Elisabeth Mangold, $\mathrm{MD}^{17}$, \\ Hans van Bokhoven, $\mathrm{PhD}^{1,18}$, Michael J. Dixon, BDS, PhD ${ }^{5}$, Tjitske Kleefstra, MD, PhD ${ }^{1,18}$, \\ Alexander Hoischen, $\mathrm{PhD}^{1,18}$ and Carine E.L. Carels DDS, PhD ${ }^{2,3}$
}

\begin{abstract}
Purpose: We aimed to identify a novel genetic cause of tooth agenesis (TA) and/or orofacial clefting (OFC) by combining whole-exome sequencing (WES) and targeted resequencing in a large cohort of TA and OFC patients.

Methods: WES was performed in two unrelated patients: one with severe TA and OFC and another with severe TA only. After deleterious mutations were identified in a gene encoding low-density lipoprotein receptor-related protein 6 (LRP6), all its exons were resequenced with molecular inversion probes in 67 patients with TA, 1,072 patients with OFC, and 706 controls.
\end{abstract}

Results: We identified a frameshift (c.4594delG, p.Cys1532fs) and a canonical splice-site mutation (c.3398-2A $>$ C, p.?) in LRP6, respectively, in the patient with $\mathrm{TA}$ and $\mathrm{OFC}$ and in the patient with severe TA only. The targeted resequencing showed significant enrichment of unique LRP6 variants in TA patients but not in nonsyndromic OFC patients. Of the five variants in patients with TA, two affected the canonical splice site and three were missense variants; all variants segregated with the dominant phenotype, and in one case the missense mutation occurred de novo.

Conclusion: Mutations in LRP6 cause TA in humans.

Genet Med advance online publication 10 March 2016

Key Words: LRP6; molecular inversion probes; tooth agenesis; Wnt/ $\beta$-catenin canonical signaling pathway

\section{INTRODUCTION}

Tooth agenesis (TA) and orofacial clefting (OFC) are distressing to families. Both are common congenital disorders and can occur as isolated entities or accompanied by other symptoms or as part of a syndrome. ${ }^{1,2}$ As an isolated condition, TA in the severe form involves six or more teeth failing to develop (oligodontia) and has a prevalence of $0.1-0.5 \%$ in populations worldwide; the frequency of the mild form of TA, which involves one

\footnotetext{
The last two authors contributed equally to this work.

The first three authors contributed equally to this work.

${ }^{1}$ Department of Human Genetics, Radboud University Medical Center, Nijmegen, The Netherlands; ${ }^{2}$ Department of Orthodontics and Craniofacial Biology, Radboud University Medical Center, Nijmegen, The Netherlands; ${ }^{3}$ Department of Oral Health Sciences, University Hospitals KU Leuven, Leuven, Belgium; ${ }^{4}$ Institute of Human Genetics, Department of Genomics, Life \& Brain Center, University of Bonn, Bonn, Germany; ${ }^{5}$ Faculty of Life Sciences and Dental School, University of Manchester, Manchester, UK; ${ }^{6}$ Department for Health Evidence, Radboud University Medical Center, Nijmegen, The Netherlands; ${ }^{7}$ Department of Molecular Developmental Biology, Radboud Institute for Molecular Life Sciences, Radboud University, Nijmegen, The Netherlands; ${ }^{8}$ DNA Facility, Center for Human Genetics, University Hospitals KU Leuven, Leuven, Belgium; ${ }^{9}$ Department of Oral and Maxillofacial Surgery, Radboud University Medical Center, Nijmegen, The Netherlands; ${ }^{10} \mathrm{Cleft}$ Lip Palate Team and AGORA-Support Group, Departments of Otorhinolaryngology and Head and Neck Surgery, Maxillofacial Surgery, and Orthodontics, University Hospitals KU Leuven, Leuven, Belgium; ${ }^{11}$ Center for Human Genetics, Department of Clinical Genetics, University Hospitals KU Leuven, Leuven, Belgium; ${ }^{12}$ Department of Molecular and Human Genetics, Baylor College of Medicine, Houston, Texas, USA; ${ }^{13}$ Human Genome Sequencing Center, Baylor College of Medicine, Houston, Texas, USA; ${ }^{14}$ Department of Pediatrics, Baylor College of Medicine, Houston, Texas, USA; ${ }^{15}$ Texas Children's Hospital, Houston, Texas, USA; ${ }^{16}$ Hubrecht Institute, KNAW and University Medical Center Utrecht, Utrecht, The Netherlands; ${ }^{17}$ Institute of Human Genetics, Biomedical Center, University of Bonn, Bonn, Germany; ${ }^{18}$ Department of Cognitive Neurosciences, Donders Institute for Brain, Cognition and Behaviour, Radboud University Medical Center, Nijmegen, The Netherlands. Correspondence: Carine Carels (carine.carels@radboudumc.nl) and Alexander Hoischen (alexander.hoischen@radboudumc.nl)
} 
to five teeth missing (hypodontia), is $3-20 \%$ of the population. ${ }^{2}$ For isolated nonsyndromic OFC, a worldwide prevalence of $0.1-0.2 \%$ has been reported. ${ }^{1}$

Although genetic heterogeneity is present for selective tooth agenesis (STHAG), including causal mutations in MSX1 (STHAG1; MIM 106600), ${ }^{3}$ PAX9 (STHAG3; MIM 604625), WNT10A (STHAG4; MIM 150400), or EDA (STHAGX1; MIM 313500), mutations of WNT10A, ligand of the Frizzled (FZD) coreceptor in the canonical WNT/ $\beta$-catenin signaling pathway, are most frequently associated with isolated TA. ${ }^{4}$ Of all genes in which mutations causing STHAG have been identified, only MSX1 has the annotation "with or without orofacial cleft," which refers to the rare combination of TA and different types of OFC in the affected members in two families identified to date. $^{5,6}$

Mutations in other genes, e.g., IRF6 (MIM 607199) and TP63 (MIM 603273), have also been shown to cause combined TA-OFC, in syndromic as well as nonsyndromic phenotypes.

Although individuals with OFC present with higher frequencies of dental anomalies, including TA in their maxillary primary and permanent dentitions, than controls, it has been suggested that the combined TA-OFC phenotype is only rarely due to genetic factors; rather, it is due to acquired disturbances of the physical environment surrounding the developing dentition. Only some families carry rare mutations in specific genes that influence both tooth development and palatogenesis, suggesting rare monogenic conditions in such cases. ${ }^{8}$

Here, we report the discovery of truncating mutations in LRP6 (MIM *603507) by whole-exome sequencing in a case with TA-OFC and a case with TA only, and we hypothesize that LRP6 mutations can cause TA, OFC, or combined TA-OFC. Through targeted resequencing, we identified five additional patients with TA harboring LRP6 mutations. To support our hypothesis, we analyzed the expression of Lrp6 in frontal sections of developing mouse heads at embryonic day 13 and embryonic day 15 (E13 and E15) using immunohistochemistry. Because mutations in TP63 have been shown to underlie the combined TA-OFC phenotype, we investigated whether Trp63 and Lrp6 function in the same molecular pathway.

\section{MATERIALS AND METHODS}

\section{Patient phenotype description}

The first index patient was a boy born with a bilateral cleft lip, a left-sided cleft of the alveolus, and a complete cleft of the hard and soft palate. He had agenesis of 4 deciduous and 17 permanent teeth. Additional features were growth retardation, hypermetropia, and a small median alveolar cleft in the mandible (Figure 1a-h). His mother also had severe TA, but not OFC, as did the maternal grandmother and her brother.

The second index patient had agenesis of two deciduous and nine permanent teeth (Figure $\mathbf{1} \mathbf{i}-\mathbf{q}$ ). Other dental features included impaction of tooth 24 and conical teeth 13 and 23. He has clinodactyly of the fifth fingers and hypermobile joints.

We obtained written consent from patients and their parents to publish their photographs (Figure 1).
For the full phenotype description and medical family history of both index patients, we refer to Supplementary Materials and Methods online.

\section{Whole-exome sequencing}

Two protocols for whole-exome sequencing and subsequent data analysis were used for the two index patients. The first one was performed through the Baylor-Hopkins Center for Mendelian Genomics using the methods previously reported; ${ }^{9}$ the second was performed in a clinical setting at the Department of Human Genetics of the Radboud University Medical Center, similar to previous reports. ${ }^{10}$

To identify additional patients with LRP6 variants, 29 in-house diagnostic exomes sequenced in patients with a range of craniofacial disorders were queried for rare $(<1 \%$ in dbSNP141), nonsynonymous, and canonical splice-site variants in LRP6.

\section{Immunohistochemistry for Lrp6 in mouse heads}

To check the expression of Lrp6 in developing teeth and palates of mice, we generated paraffin sections of E13 and E15 mouse embryo heads and stained them with antihuman LRP6 polyclonal antibody (cat. nr.orb18907, Progen, Sanbio, The Netherlands) counterstained with hematoxylin.

\section{Regulation of Lrp6 by Trp63}

We checked for Trp63 binding sites in Lrp6 using chromatin immunoprecipitation quantitative PCR analysis. To generate additional evidence for regulation of $\operatorname{Lrp} 6$ by $\operatorname{Trp} 63$, the expression of Lrp6 in facial processes of Trp63\%- embryos was compared with that of their wild-type littermates. The breeding of the genetically modified mice was performed after ethical review and in accordance with the UK Animals (Scientific Procedures) Act of 1986.

\section{Resequencing with molecular inversion probes}

To genetically test our hypothesis regarding the contribution of rare LRP6 coding variants to the etiology of TA and common OFC, we aimed to resequence all exons of LRP6 in a large patient cohort and in controls. Molecular inversion probes were used to resequence LRP6 in 67 patients with TA, ${ }^{11} 1,073$ patients with OFC, and 706 controls (CO). The cases and controls were identified as part of the sample-collection efforts from Bonn (OFC, CO), Nijmegen (OFC), and Leuven (OFC, TA) (Supplementary Table $\mathbf{S 1}$ online).

Approval from the institutional review boards involving human subjects and the ethical committees of the respective university hospitals was obtained, as was written informed consent from all individuals.

A total of 69 molecular inversion probes were designed for the coding exons of LRP6 and used for targeted enrichment as previously described, ${ }^{6}$ with minor modifications. All coding exons of LRP6 were covered, on average, 1,045 \pm 480 -fold (average \pm SD) per sample. This was comparable for cases $(1,040$ fold) and controls (1,051-fold) (Supplementary Materials and 

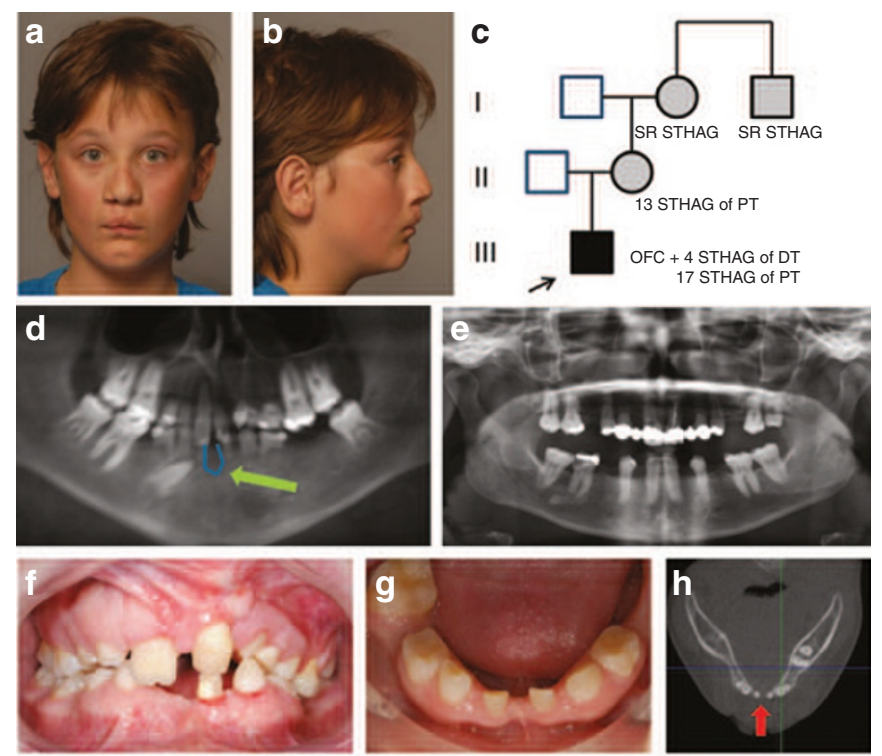
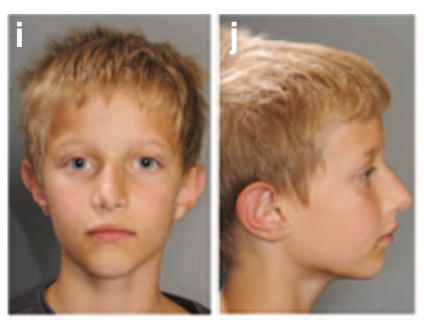

k
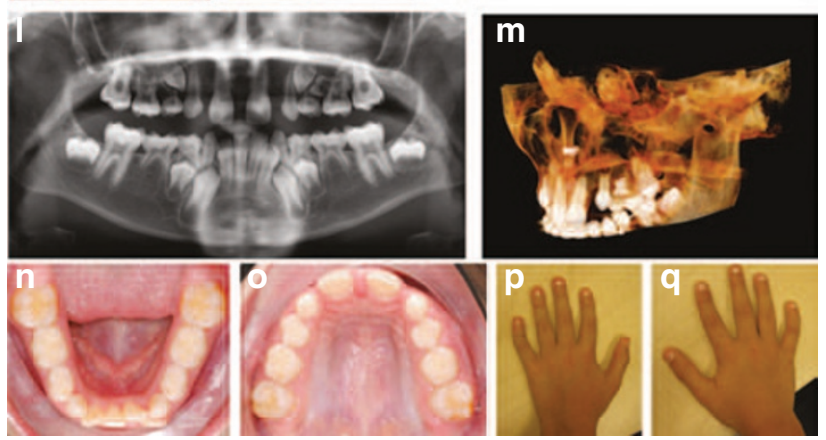

Figure 1 Clinical photographs, orthopantomogram (OPT), image from cone beam computed tomogram (CBCT), and pedigree of index patient $\mathbf{1}(\mathbf{a}-\mathbf{h})$ and index patient $\mathbf{2}(\mathbf{i}-\mathbf{q})$. ( $(\mathbf{a}$ and $\mathbf{b})$ Frontal and lateral facial photographs of index patient 1 at 12 years of age showing a repaired bilateral cleft lip with a left-sided cleft alveolus and a complete cleft of the anterior and posterior palate. He has a wide nasal base, full nasal tip, wide nasal bridge (h), and a dip in the chin (a and b). (c) His mother and maternal grandmother and her brother also have tooth agenesis (TA) but no other orofacial abnormalities. (d) The patient presented with severe TA or oligodontia; he had agenesis of four deciduous teeth $(52,62,72$, and 82 ) and was missing 18 teeth in the permanent dentition, 17 due to TA (teeth 15, 14, 13, 12, 22, 23, 24, 25, 35, 34, 33, 32, 31, 41, 42, 44, 45; excluding third molars) and 1 due to extraction (tooth 36). He had a small median mandibular cleft, which can be seen on the (d) orthopantomogram (OPT) at the green arrow, (f and $\mathbf{g})$ the intraoral photographs, and (h) the horizontal tomographic view of a CBCT. (e) The OPT of the boy's mother shows severe TA because she was missing 13 permanent teeth. (i and $\mathbf{j}$ ) Frontal and lateral photographs of index patient 2 at 9 years of age showing mild facial dysmorphic features including a narrow nasal ridge, posteriorly rotated ears with a thin helix, small earlobes, and a long superior crus antihelix. (k) He has unaffected parents and two unaffected brothers. (I) The OPT shows TA of two deciduous teeth (52 and 62) and TA of nine permanent teeth $(17,15,14,12,22,25,27,35$, and 45$)$. (I and $\mathbf{~}$ ) There is an ectopic tooth germ in the upper right molar area (tooth 17 or 18) and a horizontally impacted premolar germ (tooth 24) in the left upper quadrant. (n) The occlusal photograph of the mandibular dental arch in the mixed dentition shows malposition of tooth 32. (o) The shapes of the palatal cusps of teeth 16 and 26 are abnormal, making them resemble a second molar on the occlusal photograph of the maxillary dental arch. ( $\mathbf{p}$ and $\mathbf{q}$ ) Clinodactyly of the fifth fingers. DT, deciduous teeth; PT, permanent teeth; OFC, orofacial clefting; STHAG, selective tooth agenesis.

Methods online).The identified variants were filtered using the same filter steps for cases and controls (Table 1).

\section{RESULTS}

\section{Exome sequencing of index patients 1 and 2}

After filtering for rare coding variants found with whole-exome sequencing (Supplementary Table S2 online), we identified a deleterious frameshift (c.4594delG, p.Cys1532fs) and a canonical splice-site mutation (c.3398-2A>C, p.?) in LRP6 in index patient 1 with severe TA and OFC and in the unrelated index patient 2 with severe TA only, respectively. The frameshift mutation was predicted to cause a premature stop 15 codons downstream. Because this mutation is located in the last exon of $L R P 6$ (Supplementary Figure S1 online), the mRNA is predicted to escape nonsense-mediated decay, resulting in a truncated protein missing all terminal phosphorylation domains (PPPSP) and thereby disrupting further downstream WNT signaling. LRP6 mutants lacking two of the five PPPSP motifs are mostly inactive. ${ }^{12}$

The canonical splice-site mutation, located at a highly conserved canonical splice acceptor of intron 15, is predicted to severely disrupt splicing (Supplementary Table S2 and Figure S1 online). Based on their location, conservation, protein function, and segregation, these LRP6 mutations were considered causal for the two patients' TA phenotypes.

\section{Immunohistochemistry for Lrp6 in mouse heads}

Immunohistochemistry staining of Lrp6 on sections of the embryonic mouse heads (Supplementary Figure S2a,b online) showed that $L r p 6$ is expressed in areas of bone formation at E13 (Supplementary Figure S2a online) but more clearly expressed and including palatal bone at E15 (Supplementary Figure S2b online). Interestingly, $\operatorname{Lrp} 6$ is expressed in the tooth follicle, especially in the inner enamel epithelium (Supplementary Figure S2b online), suggesting a role for Lrp6 in early tooth development.

\section{Regulation of Lrp6 by Trp63}

The presence of a functional Trp63 binding site in intron 7 of $\operatorname{Lrp} 6$ was identified using chromatin immunoprecipitation quantitative PCR analysis (Supplementary Figure S2c online). Although Lrp6 expression is downregulated in the facial processes of Trp63-1embryos compared with their wild-type littermates, this reduction was not significant (Supplementary Figure S2d,e online). Furthermore, Lrp6 displayed reduced expression within the mesenchyme of E11.5 medial nasal processes between $\operatorname{Tr} p 63^{+/+}$and Trp63-- embryos (data not shown), but this effect was subtle. 
Table 1 Overview of $\angle R P 6$ variants identified with MIP screen in OFC and TA patients versus controls

\begin{tabular}{|c|c|c|c|c|}
\hline & $\begin{array}{c}\text { Total cases with } \\
\text { variant }(n=1,139)\end{array}$ & $\begin{array}{l}\text { TA cases with variant } \\
\qquad(n=67)\end{array}$ & $\begin{array}{c}\text { OFC cases with } \\
\text { variant }(n=1,072)\end{array}$ & $\begin{array}{c}\text { Controls with } \\
\text { variant }(n=706)\end{array}$ \\
\hline Rare $(<0.1 \%)$ coding and SS variants & 30 & 8 & 22 & 16 \\
\hline Synonymous & 3 & 1 & 2 & 3 \\
\hline Nonsynonymous & 27 & $7^{\mathrm{b}}$ & $20^{c}$ & $13^{b, c}$ \\
\hline Unique/private variants & 13 & 5 & 8 & 5 \\
\hline Synonymous & 1 & 0 & 1 & 2 \\
\hline Nonsynonymous & 12 & $5^{d}$ & $7^{e}$ & $3^{\mathrm{d}, \mathrm{e}}$ \\
\hline CADD PHRED > 20 & 7 & $4^{f}$ & $3^{9}$ & $1^{f, g}$ \\
\hline
\end{tabular}

All variants were coding or canonical splice-site variants with a population frequency of $<0.1 \%$ (based on dbSNP142 and an in-house database with $>5,000$ samples). Three or fewer samples in this study had the same variant and a "GATK quality by depth" of >1,000; the latter was based on previous MIP data and extensive validations by Sanger sequencing showing low false-positive rates and high sensitivity. Minimal average coverage over all MIPs of included samples was $100-f o l d$. Most unique and rare nonsynonymous variants reported here in cases have been validated by Sanger sequencing.

MIP, molecular inversion probe; OFC, orofacial clefting; TA, tooth agenesis.

asplice-site canonical dinucleotide; CADD PHRED-like score. ${ }^{21}$ b,cSeven rare, nonsynonymous variants in $L R P 6$ in 67 cases with OD were highly significant when compared with the variant load in 706 controls (Fisher's exact test after Bonferroni correction $P=0.0056022$ ), whereas 20 rare, nonsynonymous variants in $L R P 6$ in 1,072 cases with OFC do not show significance when compared with the variant load in 706 controls. deFive unique, nonsynonymous variants in $L R P 6$ in 67 cases with OD were highly significant when compared with three such variants in 706 controls (Fisher's exact test after Bonferroni correction $P=0.0012354$ ), whereas seven unique, nonsynonymous variants in $\angle R P 6$ in 1,072 cases with OFC do not show significance when compared with the variant load in 706 controls. f,gFour unique, predicted damaging variants in LRP6 in 67 cases with OD were highly significant when compared with one such variant in 706 controls (Fisher's exact test after Bonferroni correction $P=0.001515$ ), whereas seven unique, nonsynonymous variants in $L R P 6$ in 1,073 cases with OFC do not show significance when compared with the variant load in 706 controls.

\section{Resequencing with molecular inversion probes}

Significant enrichment of rare variants was found only in cases with TA, as compared with controls (7/67 vs. 13/706; Fisher's exact test after Bonferroni correction $P=0.0056022$ (Table 1)), with a similar significant enrichment found when considering unique variants $(5 / 67$ vs. $3 / 706 ; P=0.0012354)$ or predicted damaging variants (CADD PHRED-like score $>20$ ) (4/67 vs. $1 / 706 ; P=0.001515)$. There was no difference between cases and controls for rare or unique synonymous variants. There was no statistically significant enrichment for unique $L R P 6$ variants in OFC cases compared with controls.

All unique variants in TA cases were validated by Sanger sequencing, and segregation analysis showed segregation in all families (Supplementary Figure S3a-e online); hence all five variants were considered likely pathogenic (Supplementary Table S3a online). These contained two canonical splice-site variants as well as three missense variants. Of note, the sporadic case, TA2, showed de novo mutation. Based on the gene-specific mutation rates of Samocha et al., ${ }^{13}$ the chance of identifying a de novo missense mutation in LRP6 in 67 TA cases is extremely low ( $P=0.006076$ exact Poisson test). The ultimate proof of pathogenicity of the presented missense variants will require additional functional evidence.

Careful inspection of all medical records of the seven described cases harboring unique LRP6 mutations (Supplementary Figure S4 and Table S3b) revealed additional dental anomalies, including tooth ankylosis $(n=2)$, abnormal tooth shape $(n=2)$, enamel defects $(n=1)$, and other symptoms, such as clinodactyly $(n=3)$. Furthermore, growth hormone supplementation therapy had been considered for four of these seven patients.

Severe TA was also present in a patient carrying a rare LRP6 variant (Supplementary Figures S3f and $\mathbf{S 4 j}, \mathbf{k}$ online). This suggests that several of the rare variants may still be involved in the phenotype.

\section{DISCUSSION}

We report that loss of function of LRP6 causes TA. LRP6 is a transmembrane cell surface protein that is a member of the low-density lipoprotein receptor gene family. LRP6 acts as a coreceptor for WNT together with Frizzled (FZD), transmitting signals in the canonical WNT/ $\beta$-catenin-TCF signaling cascade, which is well known for its role in differentiation, proliferation, and migration processes during dental and orofacial development. Two genes of this pathway have already been implicated in human TA. Mutations in AXIN2 (MIM 608615) were associated with TA-colorectal cancer predisposition, and mutations in WNT10A can cause either isolated ${ }^{4}$ or syndromic TA (WNT10A; MIM 606268). Lrp6 has been described as positively regulating $\mathrm{WNT} / \beta$-catenin signaling by Wnt receptor internalization (GO:0038013) in LRP6 signalosomes. ${ }^{14}$

Our findings regarding TA further expand the spectrum of LRP6 phenotypes. Although loss- or gain-of-function mutations in LRP6 and in another closely resembling Wnt coreceptor, LRP5, had already placed the Wnt pathway central in bone biology, Wnt has emerged as an important regulator of skeletal modeling and remodeling. ${ }^{15}$ LRP6 is also key to parathyroid hormone (PTH) signaling, which regulates osteoblast activity; PTH binds to its receptor PTH1R and thereby induces the PTH-PTH1R-LRP6 protein complex, leading to increased bone formation in rats. ${ }^{16}$ Because LRP6 mutations may disrupt this PTH-PTH1R-LRP6 interaction, effects on growth could result. This could explain why growth hormone supplementation therapy was being considered for four of our seven patients with unique LRP6 mutations. Other human diseases related to LRP6 variants include atherosclerosis, ${ }^{17}$ osteoporosis, ${ }^{15}$ and metabolic syndrome. ${ }^{18}$ Recently, rare LRP6 mutations were reported in spina bifida. ${ }^{19}$

Although genetic inactivation of Lrp6 was also reported to lead to cleft lip with cleft palate in a mouse model, ${ }^{20}$ our genetic data do not support a role for LRP6 mutations in nonsyndromic 
OFC in humans. Moreover, our functional data fail to show significant regulation of Lrp6 by Trp63. Because we only found evidence for molecular underpinning of TA by LRP6 mutations, it remains unsolved whether the LRP6 mutation in our index patient with combined TA-OFC also contributed to the OFC development in this patient.

Based on our results, an estimate can be made regarding the frequency of LRP6 mutations in the normal population because frequencies of TA in general populations are known. In our cohort, 3 of 706 control individuals (0.4\%) were carriers of unique LRP6 variants. Although this frequency matches population frequencies for severe TA ranging between 0.1 and $0.5 \%$, it remains unclear whether those variants are pathogenic. We hypothesize that $L R P 6$ is a major cause of severe TA but not a cause of common TA ( 3 to $20 \%$ in populations worldwide).

In conclusion, we show that LRP6 mutations cause severe TA with or without other dental anomalies such as ankylosis, enamel defects, and tooth-shape anomalies. However, our genetic data do not provide evidence that rare monogenic LRP6 mutations underlie nonsyndromic orofacial clefts.

Note: during the preparation of this article, the following paper was published: MP Massink et al., Loss-of-function mutations in the WNT coreceptor LRP6 cause autosomal-dominant oligodontia, Am J Hum Genet; 2015;97:621-626. This study provides additional independent evidence for the causative role of LRP6 mutations in TA.

\section{SUPPLEMENTARY MATERIAL}

Supplementary material is linked to the online version of the paper at http://www.nature.com/gim

\section{ACKNOWLEDGMENTS}

The Center for Mendelian Genomics is supported by the National Institutes of Health National Human Genome Research Institute (grants RO1NS058529 and U54HG006542 to J.R.L.). Research in the Dixon Laboratory, University of Manchester, is supported by the Medical Research Council (G0901539 and MR/M012174/1 to M.D.). Support was also received from the European Orthodontic Society (048 EOS research grant, 2010 to C.C.).

The authors thank members of the Nijmegen Cleft Lip and Palate Team, R. Admiraal, W. Borstlap, T. Wagner, M. Nienhuis, V. Engels, M. Kuijpers, J. Vanderstappen, E. Kerkhofs, A.M. Kuijpers-Jagtman, M. Nijhuis, J. Buchner, and M. van der Looy. The authors also thank all the affected individuals, their parents, families, and control individuals from Bonn, Leuven, and Nijmegen who participated in this study.

\section{DISCLOSURE}

The authors declare no conflict of interest.

\section{REFERENCES}

1. Schutte BC, Murray JC. The many faces and factors of orofacial clefts. Hum Mol Genet 1999;8:1853-1859. http://www.ncbi.nlm.nih.gov/pubmed/10469837.

2. Polder BJ, Van't Hof MA, Van der Linden FP, Kuijpers-Jagtman AM. A metaanalysis of the prevalence of dental agenesis of permanent teeth. Community Dent Oral Epidemiol 2004;32:217-226.

3. OMIM. Online Mendelian Inheritance in Man. Johns Hopkins University, Center for Medical Genetics: Baltimore, MD, 1996. http://www.omim.org.

4. van den Boogaard MJ, Créton M, Bronkhorst $Y$, et al. Mutations in WNT10A are present in more than half of isolated hypodontia cases. J Med Genet 2012;49:327-331.

5. van den Boogaard MJ, Dorland M, Beemer FA, van Amstel HK. MSX1 mutation is associated with orofacial clefting and tooth agenesis in humans. Nat Genet 2000;24:342-343.

6. Liang J, Zhu L, Meng L, Chen D, Bian Z. Novel nonsense mutation in MSX1 causes tooth agenesis with cleft lip in a Chinese family. Eur J Oral Sci 2012;120:278-282.

7. Matalova E, Fleischmannova J, Sharpe PT, Tucker AS. Tooth agenesis: from molecular genetics to molecular dentistry. J Dent Res 2008;87:617-623.

8. Howe BJ, Cooper ME, Vieira AR, et al. Spectrum of dental phenotypes in nonsyndromic orofacial clefting. J Dent Res 2015;94:905-912.

9. Lupski JR, Gonzaga-Jauregui C, Yang Y, et al. Exome sequencing resolves apparent incidental findings and reveals further complexity of SH3TC2 variant alleles causing Charcot-Marie-Tooth neuropathy. Genome Med 2013;5:57

10. de Ligt J, Willemsen $\mathrm{MH}$, van Bon BW, et al. Diagnostic exome sequencing in persons with severe intellectual disability. N Engl J Med 2012;367:1921-1929.

11. Dreesen K, Swinnen S, Devriendt K, Carels C. Tooth agenesis patterns and phenotype variation in a cohort of Belgian patients with hypodontia and oligodontia clustered in 79 families with their pedigrees. Eur J Orthod 2014;36:99-106

12. MacDonald BT, Yokota C, Tamai $K$, Zeng X, He X. Wnt signal amplification via activity, cooperativity, and regulation of multiple intracellular PPPSP motifs in the Wnt co-receptor LRP6. J Bio/ Chem 2008;283:16115-16123.

13. Samocha KE, Robinson EB, Sanders SJ, et al. A framework for the interpretation of de novo mutation in human disease. Nat Genet 2014;46:944-950.

14. Bilic J, Huang YL, Davidson G, et al. Wnt induces LRP6 signalosomes and promotes dishevelled-dependent LRP6 phosphorylation. Science 2007;316:1619-1622.

15. Williams BO, Insogna KL. Where Wnts went: the exploding field of Lrp5 and Lrp6 signaling in bone. J Bone Miner Res 2009;24:171-178.

16. Wan M, Yang C, Li J, et al. Parathyroid hormone signaling through low-density lipoprotein-related protein 6. Genes Dev 2008;22:2968-2979.

17. Mani A, Radhakrishnan J, Wang H, et al. LRP6 mutation in a family with early coronary disease and metabolic risk factors. Science 2007;315:1278-1282.

18. Singh R, Smith E, Fathzadeh M, et al. Rare nonconservative LRP6 mutations are associated with metabolic syndrome. Hum Mutat 2013;34:1221-1225.

19. Lei $Y$, Fathe $K$, McCartney D, et al. Rare LRP6 variants identified in spina bifida patients. Hum Mutat 2015;36:342-349.

20. Song L, Li Y, Wang K, et al. Lrp6-mediated canonical Wnt signaling is required for lip formation and fusion. Development 2009;136:3161-3171.

21. Kircher $M$, Witten $D M$, Jain $P$, et al. A general framework for estimating the relative pathogenicity of human genetic variants. Nat Genet 2014;46: 310-315. 